\title{
Toward a complete description of nucleation and growth in liquid-liquid phase separation
}

Short title : Nucleation and growth in liquids

Jean Colombani* and Jacques Bert

Laboratoire de Physique de la Matière Condensée et Nanostructures

Université Claude Bernard Lyon 1 and CNRS

6, rue Ampère, 69622 Villeurbanne cedex, France

* Corresponding author, Jean.Colombani@lpmcn.univ-lyon1.fr

\begin{abstract}
The phase separation mechanism of a binary liquid mixture off-critically quenched in its miscibility gap is nucleation and growth, its homogeneous phase reaching a metastable equilibrium state. The successive stages of growth of the nucleated droplets are a diffusion-driven free growth, an intermediate regime and a coarsening by reduction of interface (Ostwald ripening or Brownian collisions induced coalescence). We have made light attenuation experiments to investigate the sedimentation in such systems. These results have given us access experimentally to two values predicted theoretically: the growth exponent of the intermediate regime and the crossover time between this regime and interface-reduction coarsening. These data, added to the literature results, have permitted to get a quite complete view of the growth scenario in very off-critical phase-separating liquids.
\end{abstract}

\section{Introduction}

In binary liquid mixtures exhibiting a miscibility gap, the two components experience an effective repulsive interaction. At high temperature, this repulsion is overwhelmed by thermal stirring and the system is monophasic. Under a composition-dependent temperature (the binodal line, delimiting the miscibility gap), the entropic mixing is dominated by the chemical repulsion and the liquid separates in two phases. This liquid-liquid phase separation gives the opportunity to observe the pathway of a system evolving from a nonequilibrium or metastable state toward a stable equilibrium state. Indeed when quenching the mixture from the one-phase region inside the two-phases domain, the homogeneous phase abruptly reaches:

- either a nonequilibrium state, when quenched inside the spinodal line. The phase separation mechanism is here spinodal decomposition, which is now quite understood.

- or a metastable equilibrium state, when quenched between the spinodal and binodal lines. The phase-separation process is then nucleation and growth. If the 
behaviour of the early and late stages of the growth are now well established, the intermediate stage still presents open questions that we plan to study in this work.

Unlike the case of spinodal decomposition where measurements of the evolution of the growing domain size exist over at least 7 decades of time [1], measurements of the radius $R$ of the growing droplets of the minority phase after nucleation are available only over narrower windows of time, as pointed in [2]. Nevertheless, the collection of the literature results enables to get a view of the successive processes and corresponding growth laws:

- After a generally heterogeneous nucleation [3], the droplets grow with a $R \sim t^{\frac{1}{2}}$ law [4]. This evolution corresponds to a "free growth" where the solute migrates through the bulk phase by molecular diffusion toward the nuclei [5].

- An intermediate regime of slower growth is subsequently achieved when the solutedepleted layers around the nuclei start to overlap and when the interfaces between the two phases become progressively sharp. The growth law during this stage and its domain of existence is still a matter of investigation [6].

- Afterward, the equilibrium order parameter, i.e., the final volume fraction of the minority phase, equal to the initial supersaturation $\Phi$, is attained and the coarsening proceeds through the diffusion of this order parameter via two possible mechanisms : either Ostwald ripening (small nuclei evaporate and condensate onto large ones) [7] or coalescence induced by Brownian collisions [8]. Both processes lead to a reduction of the total interface and to a $R \sim t^{\frac{1}{3}}$ law $[9]$.

- Finally, when the gravitational effects dominate, a macroscopic sharing of the two phases occurs by sedimentation.

In order to evaluate the growth exponent and the range of existence of the abovementionned intermediate regime, we have used a sedimentation-based indirect method which has given us access to the radius evolution of the droplets over several decades of time.

\section{Experiments}

Our procedure is the following. We have quenched off-critical mixtures of water-isobutyric acid (showing a consolute critical point at $T_{c}=27.05^{\circ} \mathrm{C}$ ) below the binodal line. We have then studied the nucleation, growth and sedimentation in the experimental cell with a light attenuation technique, sketched in figure 1. Figure 2 displays the evolution of the light attenuation curves (transmitted light intensity over incident light intensity) along a vertical axis in the cell as a function of time. Initially the curve is a horizontal line of strong attenuation, which originates in the scattering of light by the rapidly moving nucleating and growing droplets of submicrometer size. When sedimentation sets in, a droplet-free clarification zone appears and expands at the top of the liquid due to the 
average settling motion of all the droplets. This can be observed in the curves by a steep increase just preceding the plateau: the number density of droplets starts to decrease at the top, so the light scattering diminishes at this point. The bulk droplet-rich liquid and the clarification zone are separated by the sedimentation front, which broadens with time owing to the droplets Brownian diffusion (see figure 3).

We now focus on the onset of sedimentation at time $t_{s}$. The Péclet number Pe is the ratio of the buoyant velocity $v$ of a droplet to its diffusive velocity $D / R$ : $\mathrm{Pe}=v R / D(D$ droplet diffusivity). When the former sufficiently exceeds the latter, at $t_{s}$, the droplet begins to settle down. We will tentatively consider that this occurs when the gravity effect overwhelms the diffusive effect by one order of magnitude, i.e., for $\mathrm{Pe}_{s}=10$. The position of the first droplets experiencing $\mathrm{Pe}=\mathrm{Pe}_{s}$, i.e., the first sedimenting droplets, can be traced in figure 2 through the early evolution of $z_{s}$. The point $z_{s}$ is the intercept of the increasing curve with the plateau. In other words, $z_{s}$ represents the position of the lower sedimentation front (cf. figure 31) and its motion just at $t_{s}$ reflects the settling of the droplets experiencing $\mathrm{Pe}=\mathrm{Pe}_{s}$. The velocity of these droplets is $v_{s}=\left(d z_{s} / d t\right)_{t=t_{s}}$. Their diffusion coefficient is evaluated through a Stokes-Einstein law : $D_{s}=k_{B} T /\left(5 \pi \eta R_{s}\right)(\eta$ viscosity of the majority phase). Therefore the radius of the droplets at the beginning of sedimentation $t_{s}$, calculated in introducing the above-mentionned expressions in $\mathrm{Pe}_{s}=$ $v_{s} R_{s} / D_{s}=10$, is:

$$
R_{s}=\left(\frac{2 k_{B} T}{\pi \eta\left(d z_{s} / d t\right)_{t=t_{s}}}\right)^{\frac{1}{2}} .
$$

It should be noticed that the droplets at $t_{s}$ are at the end of the "weightless" regime of growth and just enter the sedimentation-influenced growth. Consequently, their radius at this time derives only from the sedimentation-free coarsening regimes that they have experienced until this point (diffusive growth, Ostwald ripening, ... ) [10].

To allow comparison with literature results, renormalized values are needed. The reference size in nucleation and growth mechanisms is the radius of the critical nucleus (for which the bulk free energy gain exactly compensates the surface free energy loss) $R_{c}=\alpha / \Phi$ with $\alpha$ a capillary length evaluated as $\xi^{-} / 3$ ( $\xi^{-}$is the concentration fluctuations correlation length) [5]. The reference time is the relaxation time of this critical nucleus $t_{c}=R_{c}^{2} /\left(D^{-} \Phi\right)\left(D^{-}\right.$solute diffusivity) [5].

\section{Results}

We have then plotted in figure $4\left[\tau=t_{s} / t_{c}, \rho=R_{s} / R_{c}\right]$ points for experiments of quench depths $\delta T$ ranging from 0.03 to $0.50 \mathrm{~K}$ and initial supersaturation $\Phi$ ranging from 0.3 to $5.9 \%$ vol. We note that all these experiments build a universal coarsening behaviour with a crossover time $\tau_{C O} \simeq 1 \times 10^{3}$ between two growth exponents: 0.15 followed by 0.36 $\simeq 1 / 3$. The latter obviously corresponds to the interface-reduction coarsening, but the experimental dispersion has not enabled to discriminate between Brownian coalescence and Ostwald ripening [10]. It follows that the former characterizes the intermediate regime and that $\tau_{C O}$ shares the end of the intermediate regime and the beginning of the conserved order-parameter coarsening. 
To check the consistency of this interpretation with known results, the $(\tau, \rho)$ curves of the major theoretical and experimental studies in the literature for very off-critical liquid mixtures, i.e., $\Phi \leq 6 \%$, have been added in figure 4 . The discrepancies between these results reveal incoherence so a critical survey is needed. Of the pioneering work of Wong and Knobler, only the most off-critical quenches have been drawn [9]. A crossover dynamics was not expected at this time, the experimental curves are bent and growth exponents are difficult to evaluate. The quench numbered $L$ of another study of Wong and Knobler [11], reanalysed by Siebert and Knobler [12], corresponds to a supersaturation $\Phi=4.2 \%$ and has accordingly been drawn. Their values take place at the end of the diffusive growth and the beginnning of the intermediate regime. For this part of their curve, the authors indicate a growth exponent of 0.18 , which is fairly close to ours (0.15). The outstanding results of Buil et al. obtained by an original optical method for $\Phi=2.6 \%$ give access to the very beginning of the phase separation, including the nucleation and early free growth, and have been added [13]. The results of Cumming et al., also displayed, show the $t^{1 / 2}$ coarsening law unexpectedly elapsing far beyond $\tau_{C O}$ [4]. This feature can be a consequence either of a specific kinetic behaviour of the polymer blends used by these authors or more likely of the difficulty to compute renormalized quantities in macromolecular liquids comparable to those of simpler systems (cf. a discussion of this point in [14]). Baumberger et al. also find a free growth regime for $2 \%$ $<\Phi<4 \%$ - with an exponent 0.6 - beyond $\tau_{C O}$ despite their use of exactly the same system as ours [15]. We have evaluated the Péclet number of their droplets for the studied dimensionless times and we have found $P e$ ranging between 50 and 1000. Therefore their experiments should have been performed during the sedimentation regime, which could explain their high growth exponent. Finally, Tokuyama and Enomoto have made a systematic model of the phase separation dynamics after heterogeneous nucleation, to predict the coarsening laws in the three regimes, in particular for $\Phi=1 \%$ [6]. Their intermediate regime exponent $(1 / 4)$ is slightly larger than our experimental one $(0.15)$ but their crossover time $\tau_{C O} \simeq 2 \times 10^{3}$ lies in agreement with ours $\left(1 \times 10^{3}\right)$. As they concern only homogeneous nucleation, the $\rho(\tau)$ curves of the model of Sagui et al. were not included [2].

\section{Conclusion}

By collecting the main literature results and completing them with an indirect method based on the light attenuation observation of early sedimentation, we have achieved a coherent universal description of growth in phase-separating binary liquid mixtures of low supersaturation. Some minor questions remain, like for instance the exact respective domains of existence of Ostwald ripening and Brownian coalescence as a function of time and supersaturation. Besides, the field of phase separation in complex systems presents still appealing problems, like for instance the interplay between phase separation, aggregation and conformational changes in macromolecular solutions, which show wide applications in life sciences [16]. 


\section{References}

[1] Perrot F., Guénoun P., Baumberger T., Beysens D., Garrabos Y., LeNeindre B., Nucleation and growth of tightly packed droplets in fluids, Phys. Rev. Lett., 73 (1994), 688.

[2] Sagui C., O'Gorman D.S., Grant M., Nucleation and growth: Decay of a metastable state, Phys. Rev. E, 56 (1997), R21.

[3] Buil S., Delville J.P., Ducasse A., Nucleation and early stage growth in phaseseparating liquid mixtures under weak time-dependent supersaturation, Eur. Phys. J. E, 2 (2000), 105.

[4] Cumming A., Wiltzius P., Bates F.S., Rosedale J.H., Light-scattering experiments on phase-separation dynamics in binary fluid mixtures, Phys. Rev. A, 45 (1992), 885.

[5] Langer J.S., Schwartz A.J., Kinetics of nucleation in near-critical fluids, Phys. Rev. A, 21 (1980), 948.

[6] Tokuyama M., Enomoto Y., Dynamics of crossover phenomenon in phase separating systems, Phys. Rev. Lett., 69 (1992), 312.

[7] Lifschitz I.M, Slyozov V.V., The kinetics of precipitation from supersaturated solid solutions, J. Phys. Chem. Solids, 19 (1961), 35.

[8] Siggia E.D., Late stages of spinodal decomposition in binary mixtures, Phys. Rev. A, 20 (1979), 595.

[9] Wong N.C., Knobler C.M., Light-scattering studies of phase separation in isobutyric acid + water mixtures: Hydrodynamic effects, Phys. Rev. A, 24 (1981), 3205.

[10] Colombani J., Bert J., Early sedimentation and crossover kinetics in an off-critical phase-separating liquid mixture, Phys. Rev. E, 69 (2004) 011402.

[11] Wong N.C., Knobler C.M., Light scattering studies of phase separation in isobutyric acid + water mixtures, J. Chem. Phys., 69 (1978), 725.

[12] Siebert E.D., Knobler C.M., Analysis of light-scattering measurements near a cloud point, Phys. Rev. Lett., 54 (1985), 819.

[13] Buil S., Delville J.P., Ducasse A., Early stage kinetics of phase-separating liquid mixtures, Phys. Rev. Lett., 82 (1999), 1895.

[14] Lalaude C., Delville J.P., Garrabos Y., Freysz E., Ducasse A., Scaled growth of an isolated droplet generated by laser-induced phase separation in a microemulsion Comparison with systems of the same Ising class, J. Phys. IV (Paris), 5 (1995), C3, 267. 
[15] Baumberger T., Perrot F., Beysens D., Kinetics of nucleation and growth in a near-critical mixture at rest and under a shear flow, Phys. Rev. A, 46 (1992), 7636.

[16] Manno M., Emanuele A., Martorana V., Bulone D., SanBiagio P.L., PalmaVittorelli M.B., Palma M.U., Multiple interactions between molecular and supramolecular ordering, Phys. Rev. E, 59 (1999), 2222. 


\section{List of Figures}

1 Sketch of the light attenutation experiment: A) Laser (wavelength 532 nm) L) Converging lens, F) Spatial filter (pinhole), T) Temperatureregulated chamber (thermal stability $0.01 \mathrm{~K}$ ), C) Experimental transparent cell (height $3 \mathrm{~cm}$, width $1 \mathrm{~cm}$ and optical length $0.1 \mathrm{~cm}$ ), R) Charge-coupled device camera. . . . . . . . . . . . . . . . 8 8

2 Light attenuation across the cell as a function of position $z$ and time $t$ for an initial supersaturation $\Phi=8.07 \%$ and a quench depth $\delta T=0.35 \mathrm{~K}$. 9

3 Photograph of the cell during sedimentation $50 \mathrm{~min}$. after the quench for the experiment of figure 2. . . . . . . . . . . . . . . . . 10

4 Droplet radius $\rho$ at the onset of sedimentation $\tau$ in reduced units for experiments with various $\delta T$ and $\Phi<6 \%$. Radius evolutions with time from Baumberger et al. [15], Buil et al. [13], Cumming et al. [4], Siebert and Knobler [12], Tokuyama and Enomoto [6] and Wong and Knobler [9] have also been added. . . . . . . . . . . . . . . . . . 11 


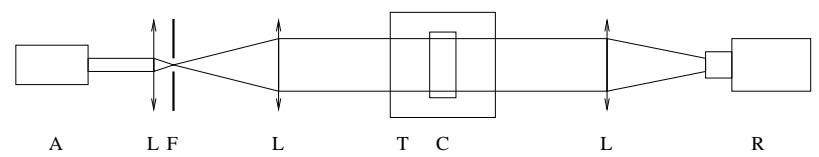

Figure 1: 




Figure 2: 


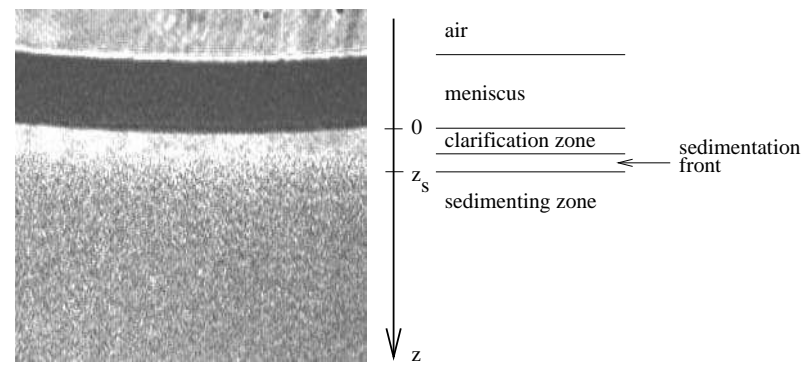

Figure 3: 


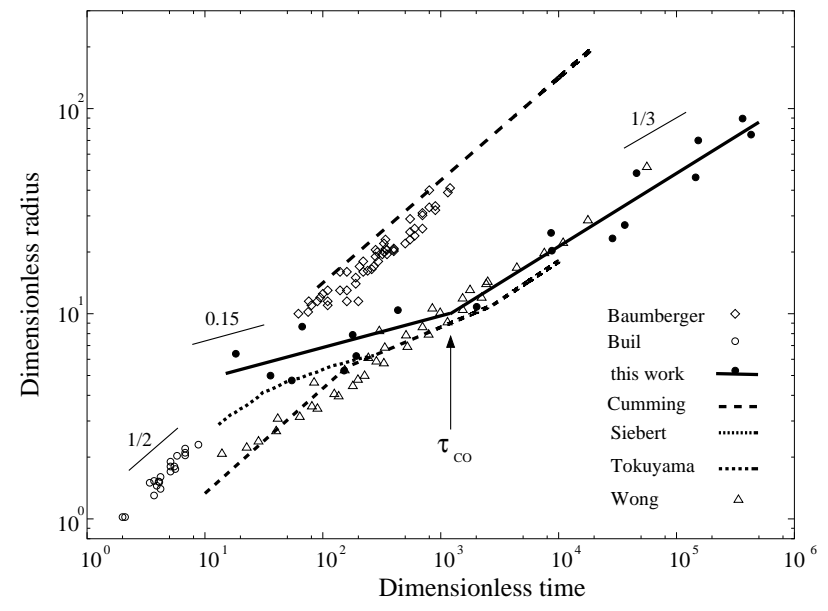

Figure 4: 